\title{
HYPOTHESIS
}

\section{Effect of pregnancy and obstructive jaundice on inflammatory diseases: the work of P S Hench revisited}

\author{
I Crocker, N Lawson, J Fletcher
}

Ann Rheum Dis 2002;61:307-310

Hench considered that cortisone improved inflammatory joint symptoms during pregnancy and obstructive jaundice. However, the improved symptoms are probably due to changes in the proportions of fatty acids in plasma and inflammatory cell phospholipids. These changes decrease the superoxide anions and eicosanoids produced and also reduce tumour necrosis factor $\alpha$ production.

\section{OBSERVATIONS OF P S HENCH}

In 1950 the Nobel Prize in physiology or medicine was awarded to Kendall, Hench, and Reichstein. Kendall and Hench were colleagues from the Mayo clinic in Rochester, USA, and Reichstein was from Basle, Switzerland. The award was for the introduction of corticosteroids in the treatment of rheumatoid arthritis. Kendall and Reichstein were chemists and their contribution was the isolation of corticosteroids, elucidation of their chemical structure and, finally, the development of semisynthetic methods for the production of isomers capable of prolonging the life of adrenalectomised animals. Hench was responsible for the use of cortisone, then known as compound E, for the treatment of chronic rheumatoid arthritis. ${ }^{1}$ The reason why Hench decided to try cortisone is not entirely clear but depended upon his interpretation of his previous observations of the circumstances in which rheumatoid arthritis improves. ${ }^{2}$

In his Heberden Oration of 1948, Hench described how impressed he was by the dramatic improvement in inflammatory joint symptoms that occurs in the majority of patients, although not all, during pregnancy or jaundice. ${ }^{2}$ He had come to the conclusion that the mechanism must be due to "a biologic compound specific in nature and function, a compound, which was normal to the human organism" and this he called "substance $X^{\prime \prime}$. In retrospect it is difficult to know why the improvement of rheumatoid arthritis during pregnancy and jaundice suggested to him that substance X might be a corticosteroid. He knew, of course, that bile acids, which are retained in obstructive jaundice, could be used as the starting material for synthesis of corticosteroids, but he had already tried the effect of bile salts on patients without success. ${ }^{3}$ He also knew, from Reichstein's work, that a corticosteroid could be converted into a substance with the biological effect of the male sex hormone because the chemical structures of testosterone and corticosteroids are similar. ${ }^{4}$ During pregnancy there is an
Accepted

6 November 2001

................... enormous increase in circulating sex hormones and an increase in the size of the adrenal glands. He also knew that minor temporary remissions in rheumatoid arthritis could be induced by procedures capable of stimulating the adrenal glands, such as general anaesthesia or surgical operation. He recognised that rheumatoid arthritis is not the only condition improved by pregnancy and jaundice, he cites fibrositis, psoriasis, and asthma, and therefore might have postulated a connection between the adrenal function and inflammation.

$$
\begin{aligned}
& \text { "The result of steroid treatment in RA was } \\
& \text { dramatic" }
\end{aligned}
$$

Whatever the reason, the result of steroid treatment was dramatic and showed that rheumatoid arthritis is not the chronic untreatable condition it was thought to be until that time, but that at least the inflammatory component could be reversed. However it is now clear that corticosteroids are not the explanation for the effect of pregnancy and jaundice and cannot be substance $X$. During obstructive jaundice corticosteroids are not significantly increased. During pregnancy cortisol certainly increases progressively, but the concentrations achieved do not affect inflammatory cell function in vitro in the way that cell function is affected by pregnancy serum. Furthermore, blood levels fall rapidly after delivery returning to normal in two to three days, which does not coincide with the pattern of rheumatoid arthritis relapse. ${ }^{56}$

If factor $\mathrm{X}$ is not cortisone then what is it? There are some clues from the observations of Hench and others. It was in 1938 that Hench published his classic account of the amelioration of rheumatoid arthritis during pregnancy. ${ }^{7}$ This was not a new observation as there had been individual case reports since the end of the previous century but Hench was the first to publish a series, 37 pregnancies in 22 patients, and he made a number of important clinical observations. The improvement starts towards the end of the first or beginning of the second trimester and progresses until term before relapse within a few weeks of delivery. Approximately $70 \%$ of patients have a clear response, which is usually greater than can be achieved with (even modern) drug treatment and if the patient responds during her first pregnancy she is likely to respond again during subsequent pregnancies. Others have since repeatedly confirmed all of these observations. ${ }^{8}$ During the 1930s and 40s Hench also reported observations on the improvement of rheumatoid arthritis during jaundice. ${ }^{39}$ Like pregnancy the effect is temporary and disappears as the jaundice 
clears. It requires quite intense jaundice either due to hepatitis or biliary obstruction but not haemolysis. Hench considered that jaundice and pregnancy shared a common mechanism, substance $X$, because the same women responded to both pregnancy and jaundice. However, the effect of jaundice was not confined to women and therefore it is unlikely to be due to female sex hormones. Unfortunately, the observations of Hench and others on the effect of jaundice have largely been forgotten so that recent work has tended to concentrate on pregnancy-specific factors. ${ }^{8}$ It is clearly necessary to look for a factor which is common to both pregnancy and jaundice and which is potent enough to produce dramatic improvement in inflamed joints.

\section{LIPID CHANGES}

One thing that pregnancy and jaundice have in common is their effect upon lipids. ${ }^{11}$ Both are associated with hypercholesterolaemia and yet the arterial lesions associated with raised cholesterol do not happen except in patients with very chronic biliary obstruction. The reason may be a change in the proportions of fatty acids in plasma phospholipids, with an increase in monounsaturated oleic acid and a decrease in polyunsaturated fatty acids, particularly arachidonic acid, thus reducing the tendency of circulating lipids to oxidise. ${ }^{12}$ Because fatty acids exchange with phospholipids at two dimensional surfaces it is their proportions in plasma which matter and not absolute concentrations. ${ }^{13}$ This is also true for the function of fatty acids in membranes as the total fatty acid composition is generally unaltered and compositional changes occur within an unchanging total lipid pool. ${ }^{14}$ Some types of inflammatory cells such as neutrophils and macrophages lack the desaturase enzyme necessary for creating further double bonds and the conversion of essential fatty acids such as linoleic acid, into less saturated fatty acids such as arachidonic acid. ${ }^{15}$ It is therefore not surprising that the proportions of fatty acids in the membranes of these inflammatory cells closely reflect the proportions of fatty acids in plasma phospholipids, although the exchange is probably through the small free fatty acid plasma pool. This exchange is rapid so that changes in plasma fatty acids are quickly reflected in membrane phospholipids, certainly within a matter of hours. ${ }^{16}$ In both jaundice and pregnancy, particularly in the third trimester, there are large changes in the proportions of fatty acids in plasma, neutrophil, and mononuclear phospholipids. These changes are much larger than can be achieved by dietary manipulation. For example, during the third trimester of pregnancy the proportion of arachidonic acid in both plasma and neutrophil phospholipids is $30 \%$ less than in nonpregnant women. ${ }^{16}$ In obstructive jaundice the changes are even greater with a reduction of between $40 \%$ and $50 \%{ }^{12}$ There is no doubt that such changes will have a profound effect upon cell function in a number of different ways.

\section{FATTY ACIDS AND INFLAMMATION}

Arachidonic acid is the precursor of inflammatory eicosanoids, including prostaglandins and leucotrienes. The rate limiting step in the cascade that produces these eicosanoids is the release of arachidonic acid from the syn 2 position of phospholipids by phospholipase $\mathrm{A}_{2}{ }^{17} \mathrm{~A}$ reduction in the proportion of phospholipid arachidonic acid means a reduction in substrate and should predict less free arachidonic acid when inflammatory cells are activated, with consequently less eicosanoid production. This prediction has been confirmed for neutrophils from patients both during jaundice and during the third trimester of pregnancy. ${ }^{12}{ }^{16}$ Women who are pregnant, compared with those who are not, show a significant reduction in arachidonic acid release from pregnancy neutrophils after stimulation with a physiological agonist (formyl peptide, fMLP) whether or not the cells were first primed by incubation with the cytokines, tumour necrosis factor $\alpha$ or interleukin 8. Similarly, there is a reduction in release of leucotriene $\mathrm{B}_{4}$, the major eicosanoid produced by neutrophils. Leucotriene $\mathrm{B}_{4}$ is an extremely potent chemotactic factor important in inflammation. Exactly the same results were obtained with neutrophils from jaundiced patients compared with cells obtained after correction of their jaundice.

One of the major functions of neutrophils is the production of superoxide radicals which are then converted into other oxygen species and play a part not only in killing of microorganisms but also in damage of surrounding tissues during uncontrolled inflammation. ${ }^{18}$ There is certainly an enormous traffic of neutrophils through rheumatoid joints, and in the synovial fluid the cells are activated to release oxygen radicals so there is little doubt that they contribute to the inflammation. ${ }^{19}$ Again during both pregnancy and jaundice the generation of superoxide radicals from activated neutrophils is significantly depressed which may explain, at least in part, the improvement in joint inflammation. ${ }^{12} 20$ The mechanism may well involve the reduction in membrane polyunsaturated fatty acids as both arachidonic acid and leucotriene $\mathrm{B}_{4}$ can stimulate the NADPH oxidase responsible for superoxide production. ${ }^{21}{ }^{22}$

\section{"During both pregnancy and jaundice the generation of superoxide radicals from activated neutrophils is} significantly depressed"

There is another and perhaps more important way in which a shift in fatty acids can affect not only enzymes such as NADPH oxidase, which is assembled within the cell membrane, but also signalling mechanisms which control cell function. This is the inevitable change in membrane fluidity that accompanies changes in the length and degree of saturation of phospholipid fatty acids. ${ }^{23}$ A reduction in the proportion of polyunsaturated and an increase in monounsaturated fatty acids will reduce cell membrane fluidity and have potentially profound effects on cell function. It should be noted that the increase in plasma cholesterol during pregnancy is not accompanied by an increase in neutrophil membrane cholesterol, which would also affect membrane fluidity (unpublished observations).

Longitudinal studies throughout pregnancy and the puerperium have shown that a reduction in the release of arachidonic acid and eicosanoids and reduced generation of superoxide anions are all detectable towards the end of the first trimester, then increase progressively during the remainder of pregnancy, and are largely reversed within six weeks of delivery. ${ }^{24}$ In other words the time course of these changes is the same as the pattern of improvement and relapse of rheumatoid arthritis during pregnancy.

It has been known for many years that plasma from women in the last trimester of pregnancy can affect inflammatory cell function and, indeed, this is one of the observations that argue against cortisone being responsible for the suppression of joint inflammation during pregnancy. ${ }^{25}$ For example, after only four hours' incubation of normal neutrophils in pregnancy plasma, arachidonic acid, leucotriene $\mathrm{B}_{4}$, and superoxide anion release are all reduced to the levels expected from pregnancy neutrophils. ${ }^{16}$ At the same time the proportions of fatty acids in cell membrane phospholipids shift dramatically so that they mimic the fatty acid content of pregnancy neutrophils. Exactly the same results are obtained when normal neutrophils are incubated in plasma from jaundiced patients. ${ }^{12}$ Furthermore, similar changes in cell function can be produced when normal neutrophil cell membrane fatty acids are exchanged by incubation in solutions of pure fatty acids as long as the fatty acids are presented in conditions which allow exchange with membrane lipid fatty acids-that is, linked to albumin, as otherwise some fatty acids such as arachidonic acid will directly stimulate neutrophils. ${ }^{26}{ }^{27}$ These are powerful 
arguments linking changes in phospholipid fatty acids with changes in inflammatory cell function that may well underlie the improvement in joint inflammation during both pregnancy and jaundice.

\section{"Powerful arguments link changes in phospholipid fatty acids with changes in inflammation"}

Possibly, when patients with rheumatoid arthritis become pregnant their cells do not respond in the same way as those from patients without an inflammatory condition. In fact, although the circulating population of neutrophils in patients with rheumatoid arthritis differs from normal, the effect of pregnancy is quantitatively the same as in normal pregnant controls so that the arguments, which can be applied to normal pregnancy, certainly apply when patients with rheumatoid arthritis are pregnant. ${ }^{24}$

\section{THE TH1/TH2 PARADIGM}

While neutrophils are important in contributing to the joint inflammation and damage of rheumatoid arthritis, present thinking about rheumatoid arthritis is that it is a Thl driven disease connected to the production of Thl associated cytokines, including tumour necrosis factor $\alpha$ and interferon $\gamma .^{28}$ Indeed immunotherapy with humanised tumour necrosis factor antibodies has been successfully used to produce temporary remissions reminiscent of those which occur in jaundice and pregnancy. ${ }^{29}$ A reduction in the proportions of polyunsaturated fatty acids affecting the availability of arachidonic acid, membrane fluidity, or both, will not only affect neutrophils but also other cells involved in inflammation-certainly macrophages and, possibly, lymphocytes. ${ }^{26}$ There is evidence that macrophage function is altered, at least during pregnancy, as when mixed mononuclear cells from the third trimester are stimulated with lipopolysaccharide, tumour necrosis factor $\alpha$ production is significantly reduced. ${ }^{30}$ This observation is in keeping with the previously reported influence on cytokine production when macrophage cell membrane fatty acids are manipulated in vitro. $^{26}{ }^{27}$ It is also in keeping with the known reduction in circulating tumour necrosis factor $\alpha$ during pregnancy. ${ }^{31}$

To return to the work of Hench, reported more than 50 years ago, it is now possible to explain the striking similarity between the effects of pregnancy and jaundice on rheumatoid arthritis. ${ }^{2}$ Both cause changes in the proportions of fatty acids in plasma and inflammatory cell membrane phospholipids associated with a reduction in inflammatory cell responses to activation. It is this change, particularly the shift away from polyunsaturated fatty acids, which is "factor X" and not the single "biologic compound, specific in nature and function" as originally envisaged, and certainly not cortisone. Figure 1 summarises this. In his Heberden Oration in 1948, Hench listed a number of conditions that he suggested may improve during pregnancy or jaundice. ${ }^{2}$ One of these is psoriasis with or without arthritis, a disease in which activated neutrophils are now known to play an important part and therefore might be expected to respond. ${ }^{32}$ Others such as asthma, hay fever, and migraine are more contentious. Pregnancy is now thought to cause a shift away from a Thl towards a Th2-type of immune response that may well be important in allowing tolerance of the antigenically foreign fetus, at least during the second and third trimesters. ${ }^{33}$ Of course the mechanism underlying the shift from Thl to Th2 is not understood. Certainly Thl driven diseases improve during pregnancy, particularly rheumatoid arthritis but also Crohn's disease and multiple sclerosis. ${ }^{34}$ All these conditions are associated with higher than normal levels of circulating tumour necrosis factor $\alpha$ which is known to be reduced during pregnancy and may be explained by changes in membrane phospholipid fatty acid saturation. Whether Crohn's disease and multiple sclerosis also improve during jaundice is not known.

\section{FATTY ACID METABOLISM}

The partial success of $\gamma$-linolenic acid supplements (evening primrose oil) for the treatment of rheumatoid arthritis appears to confirm the importance of arachidonic acid metabolism. The mechanism involves elongation of $\gamma$-linolenic acid to dihomo- $\gamma$-linolenic acid by neutrophils, but then dihomo- $\gamma$-linolenic acid cannot be converted to arachidonic acid because the cells lack the 5-desaturase enzyme. As a result dihomo- $\gamma$-linolenic acid accumulates in the cell membrane where it competes for phospholipase $\mathrm{A}_{2}$ so reducing available arachidonic acid and leucotriene $\mathrm{B}_{4}{ }^{36}$

The changes in membrane fatty acids in pregnancy and jaundice are much larger than can be produced by diet, but the modest changes resulting from increased intake of fish oils containing n-3 fatty acids aimed at reducing arachidonic acid and eicosanoid production do improve joint symptoms. ${ }^{37}$ The other situation in which fish oils or olive oil may be beneficial is in the prevention of ischaemic vascular disease. Atherosclerosis is an inflammatory disease caused by uptake of oxidised low density lipoprotein cholesterol by macrophages in vessel walls. ${ }^{38}$ A reduction in polyunsaturated fatty acids and substitution by monounsaturated acids would reduce the susceptibility of low density lipoprotein cholesterol to oxidative stress and at the same time reduce the production of inflammatory mediators by macrophages. All this emphasises the need to understand the mechanisms and manipulate them.

The first rate-limiting step in production of arachidonic acid $(20: 4, n-6)$ is the desaturation of linoleic acid $(18: 2, n-6)$ by a $\delta$ - 6 desaturase. ${ }^{39}$ This enzyme is missing from neutrophils and monocytes so that arachidonic acid in their lipid membranes depends upon uptake from plasma of arachidonic acid synthesised in the liver. ${ }^{15}$ The implication is that the

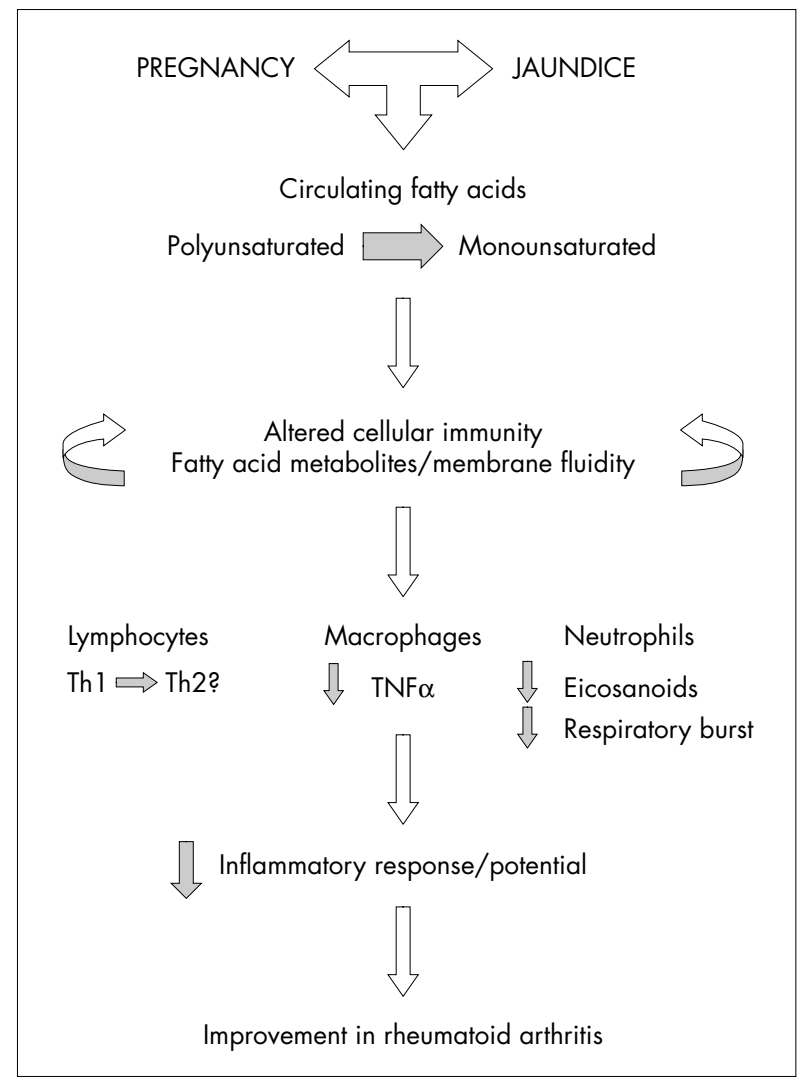

Figure 1 Fatty acid hypothesis. 
proportions of polyunsaturated fatty acids in plasma and inflammatory cell membranes depend upon hepatic desaturases. Both $\delta-6$ and $\delta-5$ desaturases of liver microsomes are under hormonal control and inhibited by oestrogens while the $\delta$-9 desaturase responsible for conversion of stearic (18:0, n-9) to oleic acid (18:1,n-9) is stimulated. ${ }^{39}$ Consequently, it seems likely that the changes in fatty acids during pregnancy relate to the enormous hormonal changes taking place. The mechanism in jaundice is uncertain but bile salts are the most likely candidates as, despite Hench's failure to show an effect when these were given either orally or intravenously, chenodeoxycholic acid has been shown to improve joint symptoms in rheumatoid arthritis. ${ }^{40}$ Whether bile salts affect desaturase activity is not known. Attempts have already been made to develop drug treatment to inhibit the hepatic $\delta-6$ desaturase, ${ }^{41}$ and now that both the human $\delta$ - 6 and $\delta-5$ desaturases have been cloned, their manipulation may become possible. $^{42} 43$

\section{Authors' affiliations}

I Crocker, J Fletcher, David Evans Medical Research Centre, Nottingham City Hospital NHS Trust, Hucknall Road, Nottingham

NG5 1PB, UK

N Lawson, Department of Clinical Chemistry, Nottingham City Hospital NHS Trust

\section{REFERENCES}

1 Hench PS, Kendall EC, Slocumb CH, Polley HF. The effect of a hormone of the adrenal cortex (17-hydroxy-1 1-dehydrocorticosterone: compound E) and of pituitary adrenocorticotrophic hormone on rheumatoid arthritis. Ann Rheum Dis 1949;8:97-104.

2 Hench PS. The potential reversibility of rheumatoid arthritis. Ann Rheum Dis 1949:8:90-6.

3 Hench PS. Effect of jaundice on rheumatoid (atrophic) arthritis and on primary fibrositis: further observations: attempts to reproduce the phenomenon. Archiv Intern Med 1938;61:451-80.

4 Reichstein T, Shoppee CW. The hormones and the adrenal cortex. In: Harris RS, Thimann KV, ed. Vitamins and hormones. New York: Academic Press, 1943:160-95.

5 Nolten WE, Lindheimer MD, Rueckert PA, Oparil S, Ehrlich EN. Diurnal patterns and regulation of cortisol secretion in pregnancy. J Clin Endocrinol Metab 1980;51:466-72.

6 Carr BR, Parker CR, Madden JD, MacDonald PC, Porter JC. Maternal plasma adreno-corticotrophin and cortisol relationships throughout human pregnancy. Am J Obstet Gynecol 1981;139:416-22.

7 Hench PS. The ameliorating effect of pregnancy on chronic atrophic (infectious rheumatoid) arthritis, fibrositis, and intermittent hydrarthrosis. Proc Staff Meet Mayo Clinic 1938;13:161-7.

8 Spector TD, Da Silva JA. Pregnancy and rheumatoid arthritis: an overview. Am J Reprod Immunol 1992;28:222-5.

9 Hench PS. The advantage of hepatic injury and jaundice in certain conditions, notably the rheumatic diseases. Med Clin North Am 1940:24:1209-37.

10 Jimenez DM, Pocovi M, Ramon-Cajal J, Romero MA, Martinez H, Grande F. Longitudinal study of plasma lipids and lipoprotein cholesterol in normal pregnancy and puerperium. Gynecol Obstet Invest 1988:25:158-64.

11 Scriven MW, Horrobin DF, Puntis MC. Study of plasma and red cell phospholipid fatty acids in extrahepatic cholestatic jaundice. Gut 1994;35:987-90.

12 Crocker IP, Lawson N, Baker PN, Fletcher J. The anti-inflammatory effects of circulating fattly acids in obstructive jaundice; similarities with pregnancy-induced immunosuppression. QJM 2001;94:475-84.

13 Holman RT, Johnson SB, Ogburn PL. Deficiency of essential fatty acids and membrane fluidity during pregnancy and lactation. Proc Natl Acad Sci USA 1991;88:4835-9.

14 Spector AA, Mathur SN, Kaduce TL, Hyman BT. Lipid nutrition and metabolism of cultured mammalian cells. Prog Lipid Res 1980;19:155-86.

15 Chilton L, Surette ME, Swan DD, Fonteh AN, Johnson MM, Chilton FH. Metabolism of gammalinolenic acid in human neutrophils. J Immunol $1996 ; 156: 2941-7$
16 Crocker I, Lawson N, Daniels I, Baker P, Fletcher J. Significance of fatty acids in pregnancy-induced immunosuppression. Clin Diagn Lab Immunol 1999;6:587-93.

17 Bingham CO, Austen KF. Phospholipase $A_{2}$ enzymes in eicosanoid generation. Proc Assoc Am Physicians 1999; 1 1 1:516-24.

18 Edwards SW. Biochemistry and physiology of the neutrophil. Cambridge University Press, 1994

19 Edwards SW, Hallett MB. Seeing the wood for the trees: the forgotten role of neutrophils in rheumatoid arthritis. Immunol Today 1997; 18:320-4.

20 Crouch SP, Crocker IP, Fletcher J. The effect of pregnancy on polymorphonuclear leukocyłe function. J Immunol 1995;155:5436-43.

21 Dana R, Malech HL, Levy R. The requirement for phospholipase $A_{2}$ for activation of the assembled NADPH oxidase in human neutrophils. Biochem J 1994;297:217-23.

22 Dewald B, Baggiolini M. Activation of NADPH oxidase in human neutrophils. Synergism between FMLP and the neutrophil products PAF and $\mathrm{LTB}_{4}$. Biochem Biophys Res Commun 1985;128:297-304.

23 Kusner DJ, Aucott JN, Franceschi D, Sarasua MM, Spagnuolo PJ, King $\mathrm{CH}$. Protease priming of neutrophil superoxide production. Effects on membrane lipid order and lateral mobility. J Biol Chem 1991;266:16465-71.

24 Crocker IP, Baker PN, Fletcher J. Neutrophil function in pregnancy and rheumatoid arthritis. Ann Rheum Dis 2000;59:555-64.

25 Persellin RH, Leibfarth JK. Studies of the effects of pregnancy serum on polymorphonuclear leukocyte functions. Arthritis Rheum polymorphonuclear

26 Flesch I, Ferber E. Effect of cellular fatty acid composition on the phospholipase $\mathrm{A}_{2}$ activity of bone marrow-derived macrophages, and their ability to induce lucigenin-dependent chemiluminescence. Biochim Biophys Acta 1986:889:6-14.

27 Calder PC, Bond JA, Harvey DJ, Gordon S, Newsholme EA. Uptake and incorporation of saturated and unsaturated fatty acids into macrophage lipids and their effect upon macrophage adhesion and phagocytosis. Biochem J 1990;269:807-14

28 Feldman M, Taylor P, Paleolog E, Brennan FM, Maini RN. Anti-TNF alpha therapy is useful in rheumatoid arthritis and Crohn's disease: analysis of the mechanism of action predicts utility in other diseases. Transplant Proc 1998;30:4126-7.

29 Elliott MJ, Maini RN, Feldmann M, Kalden JR, Antoni C, Smolen JS, et al. Randomised double-blind comparison of chimeric monoclonal antibody to tumour necrosis factor alpha (cA2) versus placebo in rheumatoid arthritis. Lancet 1994;344:1 105-10.

30 Crocker IP, Fletcher J, Baker PN. Pregnancy fatty acids and the regulation of TNF alpha. J Soc Gynecol Invest 2000;7(suppl):691

31 Argiles JM, Carbo N, Lopez-Soriano FJ. TNF and pregnancy: the paradigm of a complex interaction. Cytokine Growth Factor Rev 1997:8:181-8.

32 Dunna SF, Finlay AY. Psoriasis: improvement during and worsening after pregnancy. Br I Dermatol 1989;1 20:584

33 Raghupathy R. Th 1-type immunity is incompatible with successful pregnancy. Immunol Today 1997; 18:478-82

34 Rogers RG, Katz VL. Course of Crohn's disease during pregnancy and its effect on pregnancy outcome: a retrospective review. Am J Perinatol $1995 ; 12: 262-4$

35 Confavreux C, Hutchinson M, Hours MM, Cortinovis-Tourniaire $P$ Moreau T. Rate of pregnancy-related relapse in multiple sclerosis. Pregnancy in Multiple Sclerosis Group. N Engl J Med 1998;339:28591.

36 Chilton L, Surette ME, Swan DD, Fonteh AN, Johnson MM, Chilton FH. Metabolism of gammalinolenic acid in human neutrophils. J Immuno 1996;156:2941-7.

37 van der Tempel H, Tulleken JE, Limburg PC, Muskiet FA, van Rijswijk $\mathrm{MH}$. Effects of fish oil supplementation in rheumatoid arthritis. Ann Rheum Dis 1990;49:76-80.

38 Steinberg D. Low density lipoprotein oxidation and its pathological significance. J Biol Chem 1997;272:20963-6.

39 Brenner RR, Bernasconi AM, Garda HA. Effect of experimental diabetes on the fatty acid composition, molecular species of phosphatidyl-choline and physical properties of hepatic microsomal membranes. Prostaglandins Leukot Essent Fatty Acids 2000;63:167-76.

40 Bruusgaard A, Andersen RB. Chenodeoxycholic acid treatment of rheumatoid arthritis [letter]. Lancet 1976;i:700.

41 Obukowicz MG, Raz A, Pyla PD, Rico JG, Wendling JM, Needleman P. Identification and characterization of a novel deltab/delta5 fatty acid desaturase inhibitor as a potential anti-inflammatory agent. Biochem Pharmacol 1998;55:1045-58.

42 Cho HP, Nakamura M, Clarke SD. Cloning, expression, and fatty acid regulation of the human delta-5 desaturase. J Biol Chem 1999;274:37335-9.

43 Cho HP, Nakamura MT, Clarke SD. Cloning, expression, and nutritional regulation of the mammalian delta- 6 desaturase. J Biol Chem $1999 ; 274: 471-7$. 